\title{
Seqüência Básica na Elaboração de Protocolos de Pesquisa
}

\author{
Bráulio Luna Fo
}

São Paulo, SP

Ninguém desconhece a tremenda evolução do conhecimento humano através dos tempos. Todaviaéindiscutível que dentro as várias formas de conhecimentos - arte, música, poesia, política, teologia, etc - nenhuma teve maior influência sobre a história da humanidade do que o conhecimento científico. Não obstante este prestígio, o conhecimento científico da forma que o conhecemos hoje é uma aquisição relativamente recente. A maravilhosa jornada científica teve início no século XVI, na Inglaterra, com Francis Bacon que, primeiramente, propôs uma maneira de desenvolvê-lo, estipulando para isso - o método indutivo experimental. Posteriormente, várias outras proposições sobre o assunto foram surgindo, e hoje, dentro do campo científico, aceita-se com variações, como o principal veio do qual se origina ou desenvolve o conhecimento - o método hipotético dedutivo. Para aqueles interessados em se aprofundar neste assunto, sugiro algumas fontes de consultas nas referências bibliográficas ${ }^{1,2}$.

Podemos afirmar que a diferença fundamental entre $o$ conhecimento científico e as demais formas de conhecimento, é que o primeiro além de ser fruto de observações rigorosas, planejadas e sistemáticas, procura sempre realizar conexões entre as coisas, estabelecendo generalizações capazes de explicar de maneira cada vez mais completa os fenômenos da natureza. Mas o que, especialmente, fez com que o conhecimento científico alcançasse o status que tem, é que ele é, essencialmente, crítico e exige uma constante e ininterrupta comprovação experimental das suas afirmações. Isto o torna, antes de tudo, um conhecimento em permanente construção. Este é principal corte epistemológico que o faz quase único e, ainda hoje, cada vez mais imitado por todas as atividades humanas que procuram angariar prestígio social e cultural.

Em síntese, poderíamos afirmar que foi o aparecimento do método científico que originou esta nova e revolucionária forma de desenvolver o conhecimento, possibilitando como consequiência imediata, não apenas a explicação dos fenômenos da natureza, como também colocando-se a serviço da transformação da humanidade. Ou seja, um conhecimento que não apenas aborda a natureza como também tentainstrumentalizá-la ${ }^{3}$.

Universidade Federal do Estado de São Paulo - Escola Paulista de Medicina. Correspondência: Bráulio Luna $\mathrm{F}^{\circ}$ - UNIFESP-EPM - Rua Leandro Dupret, 317 - 04025-011 - São Paulo, SP

Recebido para publicação em 10/10/98

Aceito em 9/11/98

\section{A base lógica da pesquisa}

O método científico pressupõe que toda pesquisa começa com uma pergunta ou hipótese (fig. 1). Esta pergunta ou hipótese pode se originar de várias maneiras - desde uma simples observação casual, fortuita, como também em conseqüência de perguntas anteriores. Nesta fase, o trabalho do pesquisador é comparado com o de um artista. Isto ocorre por que não existe um método ou maneira para se originar novas perguntas ou idéias. Elas geralmente são frutos do talento individual. Naturalmente, cada nova pergunta pressupõe algum tipo de hipótese mais ou menos elaborada. De maneira que podemos afirmar que não existe olhar científico indiferente.

O passo seguinte é transformar esta pergunta numa questão pesquisável. O que quer dizer desenvolver um plano de trabalho que possibilite ao pesquisador tentar respondê-la dentro da sua realidade (recursos humanos, materiais e financeiros) e considerando o estágio do conhecimento.

Este tipo de planejamento que visa responder uma pergunta ou problema em evidência, definindo a estrutura da pesquisa, selecionando o tipo e o número de variáveis a serem estudadas, e analisando os resultados encontrados, é designado de protocolo do estudo.

O protocolo constitui passo importante na realização da pesquisa. É a transcrição do método científico à pergunta formulada pelo pesquisador. Isto ocorre porque as questões científicas são freqüentemente imprecisas, os instrumentos de medidas das variáveis são comumente não confiáveis ou não disponíveis, e as relações entre as variáveis são muitas vezes enganosas. Além disso, pode constituir um momento especial para o pesquisador aprofundar as idéias básicas do projeto, considerando os aspectos teóricos e práticos de sua operacionalidade.

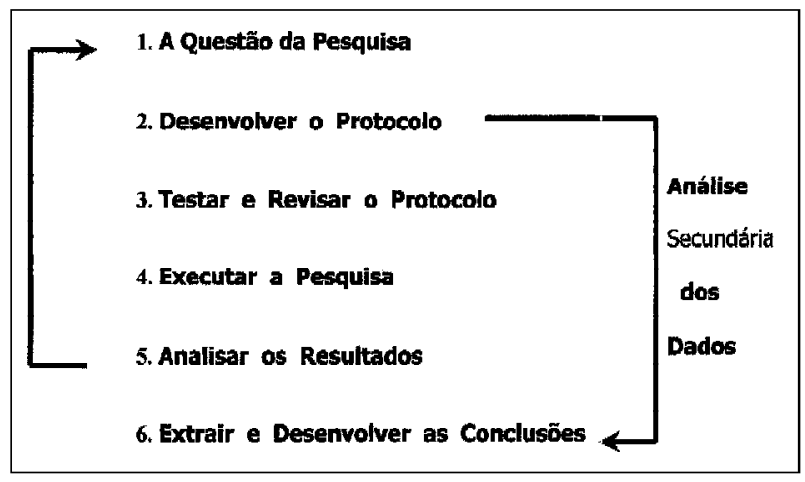

Fig. 1 - A sequiência e ciclo da pesquisa. 
É durante o planejamento da pesquisa que se deverá decidir o tipo da pesquisa, ou seja, a estrutura sobre a qual se procurará responder a pergunta: será experimental (ensaio clínico) ou será não experimental (observacional)? Uma vez decidido o tipo de desenho, deve-se considerar cuidadosamente a possibilidade de viés ou variáveis de confusão dentro do estudo, estabelecendo-se então estratégias para preveni-los ou neutralizá-los. Concomitantemente, selecionam-se também os instrumentos para as análises das variáveis em questão. Finalmente, define-se as estratégias de condução e de análises dos resultados, apontando-se para as possíveis conclusões. Muitas vezes estas conclusões originarão novas perguntas, reiniciando-se um novo ciclo da jornada científica ${ }^{4}$.

\section{O protocolo da pesquisa}

Considerando o que referimos sobre a importância do método científico, o protocolo deverá em amplas linhas contemplar os seguintes pontos: importância do tema - revisão da literatura; a questão do estudo; seleção da amostra; desenho do estudo; estratégia de condução do estudo; análise dos dados; considerações éticas; responsabilidades administrativas.

Importância do tema e revisão da literatura-Éessencial contextualizar a questão do estudo, não apenas para evitar repetição sobre temas já exaustivamente explorados na literatura, como também para sua adequação e valorização no cenário científico. A revisão da literatura poderá ajudar na melhor definição e precisão das questões pesquisáveis, facilitando o planejamento da pesquisa. Habitualmente, como o pesquisador deverá solicitar fundos dos órgãos fomentadores de pesquisa (CNPq, FAPESP, Indústrias Farmacêuticas, etc), esta parte introdutória não deve jamais ser subestimada.

A questão do estudo -Em relação às questões do estudo ou objetivos, além das considerações já enunciadas, salienta-se que podem ser divididas em primária e secundária. As questões ou objetivos primários são aquelas referentes à hipótese fundamental que originou a pesquisa. Sobre estas deverão estar voltadas todas as perguntas concernentes à formulação, desenvolvimentos do protocolo, seleção dos pacientes, tamanho da amostra, medidas das variáveis, etc. Já os objetivos secundários são aqueles que circunstancialmente poderão também ser observados, considerando a natureza da pesquisa, o tipo de desenho e a importância das variáveis acessórias na hipótese do estudo. Por exemplo, na determinação da eficácia terapêutica dos trombolíticos no tratamento do infarto agudo do miocárdio (IAM), a mortalidade global e a mortalidade cardíaca são freqüentemente avaliadas como objetivos primários; já a função do ventrículo esquerdo ou freqüência de acidentes vasculares não letais como objetivos secundários.

Dentro deste aspecto é fundamental a definição dos critérios de identificação dos casos que deverão ser sele- cionados. O investigador deve especificar as características da população alvo que melhor serve à questão da pesquisa. Isto é realizado através do estabelecimento dos critérios de inclusão e exclusão.

Critério de inclusão-Define as principais características da população alvo e acessível. É importante tomar decisões que: 1) possam ser usadas durante o estudo;2) possam ser generalizadas para outras populações; 3) caracterizem geográfica e temporalmente a população acessível, envolvendo decisões sobre objetivos práticos e científicos.

Critérios de exclusão-Indica o subgrupo de indivíduos que, embora preencha os critérios de inclusão, também apresenta características ou manifestações que podem interferir na qualidade dos dados, assim como na interpretação dos resultados. O alcoolismo aumenta a generabilidade do estudo em hipertensão arterial, mas pode ser mais difícil realizá-lo porque os pacientes são mais difíceis de seguir e aderir ao tratamento. Alguns critérios de exclusão são por considerações éticas, outros pela menor propensão de determinados pacientes em participarem do estudo. Se o número de exclusões se torna excessivo, a generalização do estudo para a população geral pode ser comprometida ${ }^{4}$.

Seleção da amostra - Outro aspecto relevante referese ao tipo de população acessível ao estudo. Neste mister há geralmente duas grandes opções.

Amostra baseada em clínica ou hospital - Tem a vantagem de ser barata e de fácil recrutamento. As desvantagens são que os fatores que determinam a vinda dos pacientes ao hospital podem ter um importante efeito sobre as características da doença em estudo. A frequiência de dor precordial como expressão de IAM nos centros terciários e quaternários é várias vezes maior que nos hospitais comunitários. Essa diferença poderá criar dificuldades na extrapolação do estudo feito em um local para outro. Por outro lado, as amostras baseadas em hospitais são boas para estudar doenças severas de curso uniforme (endocardite infecciosa, choque cardiogênico, etc).

Amostra baseada em população-Caracteriza-se pela seleção dos indivíduos em residências, produzindo amostra representativa de uma região. Sãoúteis para orientar política de saúde pública, mas tem as desvantagens de ser difícil de realizare ser dispendiosa, particularmente para as doenças raras.

Embora não seja escopo deste artigo, não poderíamos deixar de destacar que há duas grandes classes de amostras de estudo: probabilístico e não probabilístico.

Amostra probabilistica é aquela que utiliza um processo randômico para garantir que cada unidade da população tenha a mesma chance de participar no estudo. É uma abordagem científica que propicia base rigorosa para estimar a fidelidade com que o fenômeno observado na amostra seja representativo para toda a população. Permite também calcular a significância estatística.

Amostras não probabilísticas são mais práticas que as amostragens probabilísticas, mas por que os testes estatísticos são baseados na pressuposição que as amostras 
utilizadas foram probabilísticas, o objetivo de uma amostragem não probabilística é produzir um fac-símile de uma amostra probabilística para a pergunta da pesquisa. Principais tipos de amostragem não probabilística são: amostragem consecutiva e amostragem conveniente.

A amostragem consecutiva consiste em recrutar cada paciente que preencher os critérios de seleção dentro de um intervalo de tempo especificado ou número de paciente. É a melhor e a mais utilizada dentro das técnicas de amostragem não probabilística. Problema ocorre quando o período de tempo de recrutamento dos pacientes é curto demais para permitir uma amostragem representativa, ou para captar variações relacionadas com o período do ano, e que pode ser importante para a pesquisa. Por exemplo, investigação sobre doenças das vias aéreas superiores realizadas em junho podem não ser representativas de síndromes virais para o ano inteiro.

A amostragem conveniente, por sua vez, consiste na utilização daqueles pacientes disponíveis da população acessível.É amplamente empregada na pesquisa clínica devido a vantagens logísticas e custos. Apresenta como desvantagem a possibilidade de selecionar indivíduos não representativos da população alvo. No estudo MRFIT, os pacientes de alto risco foram selecionados através de anúncio; como esses voluntários eram mais sadios que o esperado ocorreram menos eventos (mortes, infarto, etc), repercutindo, na análise final dos resultados obtidos.

Deve ser evidente que aquelas pesquisas constituídas por amostras de pacientes baseadas em hospital ou em amostra não probabilística não têm, em princípio, capacidade de generalização, além da população que as originaram. Portanto, devem ser interpretadas com muito cuidado pelos pesquisadores ou médicos em outras localidades. A sua correta valorização dependerá do tipo de doença, pacientes e espectro clínico da doença observada, ao lado de dados conhecidos da literatura que respaldem os resultados encontrados nessas pesquisas ${ }^{4}$.

Tamanho da amostra - À medida que as questões acima forem sendo definidas, o pesquisador deve procurar estimar o tamanho da amostra populacional necessária para a realização do trabalho. Neste momento, o mesmo deve não apenas especificar quais os pressupostos básicos utilizados nesta estimação estatística - erro tipo I, erro tipo II, estimação da diferença entre os grupos estudados, variância das medidas realizadas, etc - como também avaliar se o tamanho proposto da amostra é factível para a realidade clínica e orçamentária da pesquisa. Não raro o número de pacientes ultrapassa a expectativa inicial de realização do trabalho, tornando inútil qualquer tentativa de realizá-lo, em conseqüência da impossibilidade de reunir o número necessário de pacientes. Os resultados obtidos com números menores não serão confiáveis, porque poderão ser fruto apenas de chance. Em dúvida sobre o que fazer, deve-se consultar o estatístico ou pessoa de maior experiência na decisão final. Às vezes é preciso reorientar as perguntas da pesquisa ou mudar o instrumento de mensuração das variáveis em estudo ${ }^{5}$.

\section{Desenho do estudo}

A estrutura designada de desenho do estudo, embora complexa, é essencial ao bom desenvolvimento do trabalho científico. A definição do tipo de desenho escolhido pressupõe uma série de decisões importantes, todas elas relacionadas com o tipo de perguntas, amostra disponível e tipo de variáveis a serem estudadas. O desenho de uma pesquisa também é utilizado na tentativa de minimizar erros (viés) que possam influenciar a opinião do investigador; além disso, sabe-se que cada tipo desenho apresenta maior ou menor propensão para algum tipo de erro. Assim, uma pessoa que não é familizarizada com os princípios do desenho de pesquisa poderá desconsiderar problemas inerentes a cada tipo dos desenhos.

Basicamente podemos classificar os tipo de desenhos em dois grandes grupos: experimental e observacional. Pela definição do tipo de eventos investigados - por exemplo ocorridos no passado -, o estudo poderá ser classificado como retrospectivo. Alternativamente, se os pacientes foram seguidos na expectativa de identificar eventos que não estavam presentes no início - o estudo será prospectivo. Sumariamente caracterizaremos os principais tipos de estudos mais empregados em cardiologia:

Estudo experimental é o tipo de investigação em que o pesquisador, desejando estudar os efeitos da exposição ou da ausência de um determinado fator, decide, ele mesmo, quais os elementos (pessoas, animais, cidades etc.) serão expostos ou não ao referido fator. Portanto são estudos em que há uma intervenção deliberada do investigador. Quando compara indivíduos expostos com outros não expostos ao referido fator, estará conduzindo um experimento controlado.

O ensaio clínico é a tentativa de tradução do estudo experimental, realizado em laboratório com animais de experimentação, para a prática clínica, utilizando pessoas ou pacientes como objeto de investigação. A seleção dos pacientes deve ser feita de forma que minimize variações que possam afetar as comparações entre os grupos em investigação. Isto é feito, geralmente, utilizando o processo de randomização. Vale apenas destacar que o ensaio clínico fornece as mais fortes evidências para o estabelecimento de relações de causalidade.

Estudos observacionais são investigações em que a informação é sistematicamente colhida, mas o método experimental não é utilizado, porque não há uma intervenção ativa do investigador. Observações sistemáticas, especialmente ao longo do tempo, podem permitir conclusão causal, e por conseguinte deve-se respeitar a eficácia desses desenhos. Os estudos observacionais podem ser: descritivos e analíticos.

Estudos observacionais descritivos caracterizam-sepor descrever uma situação. Isto é, a distribuição da doença na população em relação ao sexo, idadeou outras características.

Estudos observacionais analíticos tentam explicar uma situação ou seus processos determinativos (Por que a doença ocorre nessas pessoas? Pode a queda da incidência de uma doença ser atribuída à introdução de alguma medida 
preventiva?) É geralmente feito pela formulação de hipóteses. Eles comumente são baseados em estudos descritivos prévios.

Os estudos observacionais, descritivos e analíticos, podem ser categorizados em transversal (cross-sectional), longitudinal (cohort) e tipo caso-controle.

Estudo transversal (estudo de prevalência) fornece uma informação limitada no tempo - pontual - de uma situação. As medidas ou coletas dos dados são realizadas uma única veze no mesmo intervalo de tempo.

Estudo longitudinal fornece dados acerca de eventos ou mudanças que ocorrem em determinado espaço de tempo. As medidas ou coletas dos dados são realizadas mais de uma vez e em período de tempo diferente. O estudo longitudinal em que grupo de indivíduos é acompanhado por algum tempo é chamado de estudo coorte.

Estudo de caso-controle é o tipo de desenho em que se investiga a associação entre a ocorrência de uma doença e a exposição a algum fator suspeito daquela doença. Nesse estudo identifica-se, inicialmente, um grupo de indivíduos com (casos), e sem doença (controle). Depois se investiga no passado causas de diferenças entre as variáveis preditivas que possam explicar por quê os casos adoeceram e os controles não.

Embora exista o conceito disseminado que o ensaio clínico controlado randomizado e o estudo coorte prospectivo sejam, respectivamente, os dois melhores tipos de estudos em suas modalidades - o primeiro experimental, o segundo observacional -, nem sempre é possível aplicá-los em todas as questões científicas, cabendo aos pesquisador, em última análise, a definição do melhor tipo de desenho no estudo que pretende realizar.

\section{Estratégia da condução do estudo}

A identificação dos pacientes e das variáveis a serem mensuradas são partes centrais na elaboração do protocolo. Sobre o primeiro já falamos, abordaremos agora o segundo ponto. É extremamente importante que a escolha das variáveis reflitam, de maneira inconteste, o fenômeno de interesse. Não muito raro isto nem sempre é possível, e o pesquisador é obrigado a se utilizar de variáveis alternativas ( proxy variable). Esta possibilidade deve ser criteriosa, porque muitas vezes poderá distorcer os objetivos iniciais da investigação. Por exemplo, aceitar a história de dor precordial e eletrocardiograma como diagnóstico do IAM prévio poderá subestimar a freqüência do mesmo na população, pela impossibilidade de realizar cineangiocoronariografia em todos os indivíduos selecionados. Portanto, a definição do tipo de variáveis e como serão mensurados são pontos críticos na elaboração desta seção do protocolo.

Também quando se planeja realizar várias medidas em intervalos regulares, utilizando-se de exames laboratoriais e clínicos, é crucial que haja preocupação com a sua padronização, garantindo tanto a sua reprodutibilidade como a sua precisão. Isto deve ser realizado descrevendo, pormenorizadamente, a seqüência dos procedimentos e ministrando treinamentos nas pessoas responsáveis pelas medidas. Dependendo da dimensão do estudo, ou se o estudo envolve vários centros, deve-se definir uma estratégia para garantir o controle de qualidade das mensurações. Com este desiderato, é habitual descrever todas as questões referente ao tema, colocando-as no final do protocolo como anexo.

Tanto para os estudos observacionais como para os ensaios clínicos, recomenda-se que os pacientes e os indivíduos responsáveis pela colheita dos dados desconheçam a hipótese da pesquisa, ou não saibam o diagnóstico ou tratamento recebido pelos pacientes.

Em relação aos estudos em que se avaliam novas formas de tratamento é também necessário discriminar a estratégia de distribuição dos pacientes nos diversos grupos de preferência aleatória -, além de especificar o tipo e as doses dos medicamentos.

Nesta parte do protocolo, também se procura antever os principais problemas que podem acontecer na condução do estudo, tentando antecipar medidas de orientação e correção das mesmas. Por exemplo, perda de acompanhamento dos pacientes, não aderência ao tratamento, resultados inesperados no tratamento, etc ${ }^{6}$.

\section{Análise dos dados}

Uma das partes menos consideradas na elaboração do protocolo é a da colheita e preparação da análise dos dados. A maneira como os dados serão armazenados e classificados ajudará a avaliar os problemas circunstanciais que podem ocorrer durante a investigação: informações incompletas, exames com resultados discrepantes, perdas de seguimentos, resultados excessivamente ruins de um dos tratamentos, etc. Logicamente, um armazenamento adequado, por exemplo em banco de dados computadorizado, facilitará a análise após o término da fase de colheita dos dados. Recomenda-se que seja feito um esforço de antecipação de como os dados deverão ser analisados. A rotina inicial consiste na análise descritiva das variáveis. Em seguida uma previsão das possíveis associações de variáveis que deverão ser contempladas. É evidente que se deve realizar com moderação.

Neste momento considera-se, de acordo com o tipo de estudo e das variáveis empregadas (categóricas, dicotômicas, contínuas, com distribuição normal ou não) quais testes estatísticos poderão ser utilizados, especificando-se o nível de significância que os resultados deverão alcançar para serem considerados estatisticamente significantes. Em geral aceita-se um $p<0,05$ ou 0,01 (erro tipo I).

Todas as análises propostas nesta fase devem expressar a possibilidade que os dados obtidos poderão responder as perguntas iniciais da pesquisa. Um dos objetivos de qualquer trabalho científico é que os resultados sejam válidos para os participantes do estudo. Isto denomina-se de validade interna. Esta depende, dentre outras coisas, da qualidade do estudo e se as variáveis escolhidas representarão adequadamente o fenômeno que se pretende estudar, além do que se serão medidas de forma adequada. Paralelamente, considera-se também a validade externa quando os 
resultados do estudo, tendo validade interna, podem ser generalizados para outros indivíduos da população não participante do estudo.

Nesta circunstância, a variável estudada deve ser representativa do fenômeno investigado e a amostra que participou do estudo deve também ser representativa da população que a originou. Não devemos esquecer que as medidas de uma amostra populacional são dependentes de duas fontes de erros: erro de amostragem e erro de mensurações. Um dos objetivos do protocolo é trazê-los para os níveis mais baixo possível $(\mathrm{p}<0,05)$. Não podemos esquecer, todavia, que não obstante todas as nossas tentativas e melhor técnica é sempre possível que os resultados de um único estudo não sejam verdadeiramente representativos do fenômeno estudado ${ }^{4,6}$.

\section{Considerações éticas}

Preocupações com o padrão ético da pesquisa devem perpassar em todos os momentos da elaboração do protocolo. Além da necessidade que o projeto de pesquisa esteja de acordo com as normas internacionais, estipuladas na Declaração de Helsinki pela Associação Médica Mundial e revisada em 1975, deve também contemplar as disposições nacionais de cada país. Recentemente, em 1997, foi promulgada no Brasil norma específica sobre o tema. Assim, segundo a legislação brasileira existe a obrigatoriedade do con- sentimento informado dos pacientes, além disso o projeto deverá ser analisado por uma Comissão de Ética Interdisciplinar de uma instituição de pesquisa. O objetivo precípuo é resguardar, em todas as fases de desenvolvimento da pesquisa, os direitos dos pacientes e evitar desvios éticos na conduta científica. Acima de qualquer interesse coletivo, que respalde um projeto de pesquisa, fica garantido o direito individual dos participantes.

\section{Responsabilidades administrativas}

A responsabilidade do(s) pesquisador(es) deve ser clara, inclusive com descrição de cada participação, dos recursos materiais, laboratoriais e humanos previstos no projeto. Também, deve-se estimar a duração de cada etapa do projeto assim como o período do seu término. Naturalmente, dependendo das circunstâncias há necessidade de ser flexível nesta estimativa. Quando são solicitados recursos financeiros a órgãos financiadores de pesquisas, geralmente são apresentados formulários que facilitam as especificações e justificativas das demandas. Todavia é desejável a realização de estimativa pormenorizada do orçamento global do projeto.

\section{Conclusão}

Finalizando um bom projeto de pesquisa apresenta alguns atributos (Fig. 2). Primeiro é a qualidade científica do

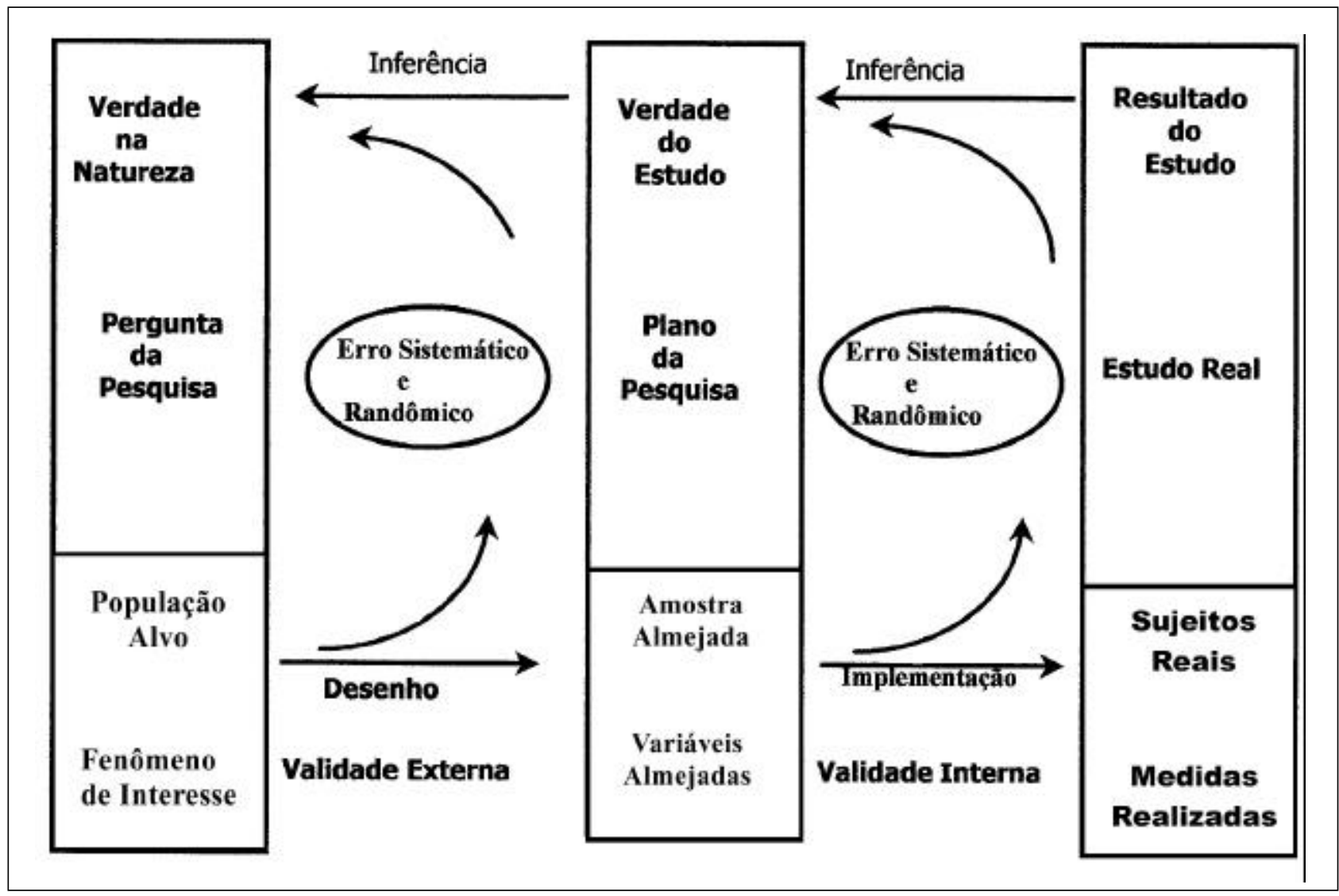

Fig. 2 - Como funciona o trabalho de pesquisa. 
plano de trabalho: basear-se em uma boa questão, usar desenho e métodos de pesquisa rigorosos e acessíveis, além de ter um grupo de pessoas experientes para realizá-lo. O segundo atributo é qualidade técnica do protocolo. Este deve ser claro, conciso, não se perdendo em detalhes periféricos do tópico, além de ser bem escrito e organizado. Quando se pretende obter financiamento, deve ser redigido levando-se em consideração que será revisto por alguém. Por conseguinte deve ser escrito com realismo, sem exagero da importância da proposta, mas considerando os aspectos positivos e negativos da mesma, valorizando, no entanto, as opções e decisões a serem realizadas. A apresentação global do projeto deverá ser formal, mas agradável e de fácil apreensão pelos revisores. Por fim, revise e revise o projeto. Quando achar que está bom, solicite ao colega mais experiente que o revise também. É sempre possível melhorar um protocolo de pesquisa, mas, muito mais complicado, se não impossível, retificá-lo quando a pesquisa já está em andamento ou em fase de análise.

"A diferença entre o conceito de saber e o de estar certo não é de grande importância, exceto quando "eu sei” pretende significar: não posso estar errado"

Ludwig Wittgenstein (On certainty)

\section{Referências}

1. Popper KR - Objective Knowledge - An Evolutionary Approach. Oxford: Oxford University Press, 1989: 385.

2. Kuhn TS - The Structure of Scientific Revolutions. $2^{\text {nd }}$ ed. Chicago: The University Chicago, 1996: 210

3. Chalmers AF-Qué Es Esa Cosa Llamada Ciencia? Una Valorization de la Naturaleza y el Estatuto de la ciencia y Sus Métodos. 15ª ed. Madrid: Siglo Veintiuno, 1994: 245.
4. Friedman LM, Furberg CD, DeMets DL - Fundamentals of Clinical Trials, $3^{\text {rd }}$ ed. St. Louis: Mosby-Year Book, 1996: 360.

5. Gehlbach SH - Interpreting the Medical Literature - Pratical Epidemiology for Clinicians. $2^{\text {nd }}$ ed. New York: Macmillan Publishing, 1998: 218.

6. Feinstein AR - Clinical Epidemiology - Architecture of Clinical Research. Philadelphia: WB Saunders, 1985: 812. 